\title{
Gods or Monsters? Non-Explicit Consent in the hastening of deaths by Intensivists in Belgium
}

\author{
Shanthi Van Zeebroeck ${ }^{a^{*}}$ \\ ${ }^{a}$ Université Libre de Bruxelles, Centre de Recherches Interdisciplinaires en Bioéthique (CRIBS), Avenue Franklin Roosevelt 50, 1050 \\ Bruxelles, Belgium.
}

Received 26 May 2019; Accepted 21 August 2019

\begin{abstract}
The Belgian Euthanasia Act of 2002 (The Act), amended in 2014 to include the Minor Act (The Minor Act), has drawn international criticisms for its liberal laws and practices regarding Euthanasia. This research study is a response to media allegations that the liberal laws on euthanasia has encouraged doctors to adopt a paternalistic approach towards their patients by terminating their lives without their explicit consent, i.e. engaging in involuntary Euthanasia. Although in theory, only voluntary euthanasia (explicit patient request and therefore consent) is permitted in Belgium, the media allegations implied that in practice, involuntary euthanasia (no explicit patient request and therefore no consent) is practiced, especially in the Intensive Care Units (ICUs) in Belgium. One major criticism is that because of its liberal laws, Belgian doctors are killing patients without their non-explicit consent. Specifically, it is alleged that Intensivists are shortening lives or hastening the deaths of their patients without their non-explicit consent in the ICUs in the Wallonia Region in Belgium. This research study conducted an empirical-qualitative study to discover if these media allegations were true or false, by interviewing heads of ICUs in five major hospitals in the Wallonia region in Belgium. The research discovered that the media allegations are true, but they are also false. The media allegations are true because shortening life or hastening the death is sometimes practiced in the ICUs without the patient's non-explicit consent. The media allegations are false because consent is not available due to the patient's critical condition, and not because it was not asked for. In other words, what is practiced in the ICUs is non-voluntary euthanasia or where patient is unable to request or consent to euthanasia.
\end{abstract}

Keywords: Euthanasia; Terminal Sedation; Intensivists; Consent; Hastening Deaths.

\section{Introduction}

Belgium becomes the most liberal of all countries in the world in its Euthanasia practices. The Belgian Euthanasia Act of 2002, (The Act), decriminalizes the practice of voluntary euthanasia in Belgium [1,2]. It grants legal authorization to a patient to request and therefore consent to be euthanized by a doctor, if the former is suffering from unbearable mental or physical pain, with no hope of recovery, and where death is imminent, and finally, if certain legal, medical, and ethical requirements are met. Concurrently, it decriminalizes the practice of voluntary euthanasia by a doctor, to depart from the transcendental "for the benefit of the patient" role of the Hippocratic doctor to become a 'for hire to either cure or to kill' role of the pre-Hippocratic doctor [3, 4]. The Act was amended in 2014 to include the Minor Act (The Minor Act), which removes all age requirements for minors. With this amendment, the Belgian euthanasia law becomes the most liberal law in the world [5].

Although in legal theory, only voluntary euthanasia (explicit patient request and therefore consent) is permitted in Belgium, media allegations implied that in practice, involuntary euthanasia (no explicit patient request and therefore

* Corresponding author: drshanthi@protonmail.com

dol http://dx.doi.org/10.28991/SciMedJ-2019-0103-3

$>$ This is an open access article under the CC-BY license (https://creativecommons.org/licenses/by/4.0/).

(C) Authors retain all copyrights. 
consent is given) is practiced, especially in the ICUs in Belgium [6]. While there were many articles of interest, two articles of interest will be mentioned in this research study. The first article made references to a second article and claimed that The Belgian Society of Intensive Care Council was administering sedative agents with the direct intention of shortening lives or hastening the deaths of patients without their non-explicit consent [7].

\section{Articles of Interest}

The first article was published in 2015 in the Journal of Medical Ethics, titled: First do no harm: Intentionally shortening lives of patients without their explicit request in Belgium [8]. And the second article was published in 2014 in the Journal of Critical Care, titled: "Piece" of mind: End of life in the intensive care unit. Statement of the Belgian Society of Intensive Care Medicine [9].

In the first article, Dr. Almagor made very important observations for this study's research. First, only voluntary euthanasia is decriminalized (legal if certain legal guidelines are met) in Belgium. Hence, involuntary euthanasia, or "the deliberate ending of life without the patient's explicit request" is prohibited. Second, based on research data conducted in the Flanders region in Belgium, he concluded that paternalistic doctors were shortening lives or hastening deaths, of their patients without their explicit request. He made references to specific statements made in the second article, where intensivists Dr. Vincent et al claimed that they do shorten lives or hasten the deaths of patients, without their explicit request, to maintain the quality of the dying process of the patient:

"Shortening the dying process with use of medication, such as analgesics/sedatives, may sometimes be appropriate, even in the absence of discomfort, and can actually improve the quality of dying; this approach can also help relatives accompany their loved one through the dying process - such a decision should be made with due consideration for the wishes of family members."

\subsection{New data}

It is important to mention that in an earlier article, Dr. Almagor noted that while there was an abundance of data in the Flanders region on non-explicit consent and the role of the doctors, in the practice of euthanasia in Belgium, there was "a need for data" in the Wallonia region [10], Hence, this research will be the very first of its kind to have conducted interviews, and to have gathered and compiled responses, from five head intensivists from five major hospitals in the Wallonia region [11].

\section{Research Objective}

\section{Are media allegations of shortening lives or hastening the deaths of patients without non-explicit consent true} or false?

The research inquiry was unequivocally precise. It was to understand whether media allegations of shortening lives or hastening the deaths of patients, without their non-explicit consent, by Intensivists in the ICUs in the Wallonia region in Belgium, true or false.

These media allegations, if proven to be true, would mean that doctors in Belgium are killing patients, which is in the eyes of the law, Murder, punishable by life imprisonment ${ }^{*}$.

\section{Research Methodology}

\section{Empirical-Qualitative Research}

An in-depth observation of the head intensivists' personal opinions about the media allegations, their personal narration of daily medical practices in the ICUs, and their personal responses to a structured questionnaire were necessary in order to meet the research objective of whether the media allegations were in fact true or false. As such, the research was empirical-qualitative in nature.

\section{Sample Size and Data Saturation}

As this was a qualitative research, the selection criteria did not depend on the quantity but the quality, i.e., the number of participants needed "to inform fully all-important elements of" the media allegations being studied. The sample size here was sufficient as it was representative of an entire medical team in each ICU of the five major hospitals in the Wallonia region, and any additional participant, therefore, would have resulted in data saturation [12].

\section{Sample selection criteria}

In conducting the research, it was quintessential to interview participants who were representative and the specific

\footnotetext{
* Under Article 14 of the Belgian Constitution, Murder, is punishable by life imprisonment.
} 
focus of the media allegations, that is, intensivists who may be hastening deaths of patients without their explicit consent. Here, the head intensivists of 5 major hospitals in the Wallonia region in Belgium satisfied this participant criteria.

\section{Procedure}

Permission to interview the head intensivists. Each head Intensivist of ICUs across five major hospitals in the Wallonia region in Belgium, namely Hospital Erasmus (Brussels), Chirec (Braine L'Alleud/Waterloo), CHU de Liège (Liège), CHU de Charleroi (Charleroi) and Le CHR de Namur (Namur), was contacted by email and permissions were granted for personal one on one interviews.

\section{Ethical considerations}

\section{Compliance with the ICU Medical Consensus Protocol (ICUMCP)*}

The research was subject to certain ethical restrictions since it concerned an ICU setting and with the head intensivists. The head intensivists made it very clear that since medical consensus is quintessential to making end of life decisions in the ICUs, it is not ethical to interview each and every intensivist, patients and/or their families. ${ }^{*}$ This will be against the ICUMCP. After pre-screening interviews and discussions with two of the leading head intensivists, the research proposal was given permission to conduct in-depth interviews as long as it was conducted exclusively with the head intensivists in the 5 major hospitals in the Wallonia region. The research was, therefore, in compliance with the ICUMCP.

\section{Data Collection method and tools}

A one-on-one in-depth interview was used to conduct the research. The interviews were conducted, and the resulting verbatim responses transcribed exclusively by the researcher. The interviews were conducted at the ICU of each head intensivist.

The type of tool used to conduct the in-depth interview was a structured questionnaire that consisted of 20 questions. The most important questions centered on media allegations of abuse, shortening life or hastening death of patients, and patient's explicit or implicit consent to shortening life or hastening death. Each interview lasted on average of approximately two hours. The longest interview recorded was 150 minutes or two-and-half hours. The responses to all 20 questions by each head intensivists were recorded by handwritten notes. To ensure the accuracy and quality of the responses, the questions and related responses were then typewritten in word format and sent to each head intensivist for verification. Once the final drafts were approved by each head intensivist, it was ready for data analysis.

\section{Data Analysis}

The type of analysis employed by the research was content analysis. Data gathered from the interviews were categorized by themes and sub-themes and simplified using a Bar Chart-Question-Report-Summary path to provide a structured documentation of the interviews.

Bar charts were used to visually display the responses of each head intensivist, on a scale of 1-4, with the numeric 1 indicating "strongly disagree" and the numeric 4 indicating "strongly agree" to each question. Under each bar chart, a question corresponding to the bar chart was followed by a report which consisted of the response from each head intensivist to the question to ensure effective representation of the bar chart display of that specific question. A summary of the responses provided an overview of how positive or negative the response was. This data analysis path was repeated for each of the 20 questions. Finally, a discussion section analyzed the collective responses of each head intensivist to each question to answer the research inquiry, namely, whether media allegations of shortening lives or hastening the deaths of patients without non-explicit consent, was true or false.

\section{Results}

\section{Question regarding media allegations}

\footnotetext{
* Please note that this research was approved by the intensivists in Belgium as satisfying "data saturation" because it was conducted specifically in the region of Wallonia, where only five hospitals are considered major hospitals. The head intensivist's opinion was representative of all the intensivists in that particular hospital's ICU. Please also note that the author was not allowed to interview any other intensivist due to ICU protocol concerning medical consensus. No other intensivist's opinions and responses is going to conflict with the head intensivist's opinion or responses. Finally, the conclusions were made based on the opinions and the responses of the head intensivists, and not based on the author's opinion.
} 
The question was whether media articles claiming that doctors in Belgium were killing their patients without nonexplicit consent true or false. It was made clear that media allegations referred specifically to ICU doctors (or intensivists). The two articles of interest were shown to each intensivist.

\section{Responses}

According to intensivist A, the media allegations were true because consent is unavailable due to the critical condition of the patient. He explained that where patient is capable of giving consent, the medical team will always respect that and will comply with the patient's wishes.

Intensivist B agreed with intensivist A regarding the willingness of the medical team to consult the patient on consent, where it is available but that in the majority of the cases in the ICU, the unavailability of consent is always an issue.

According to intensivist $\mathrm{C}$, consent is a priority as it is about patient autonomy.

According to intensivist D, the media allegations were an accurate portrayal because ICU patients, in the majority of cases, are incapable of consent.

Finally, according to intensivist E, the media allegations were true because there is no way for the patients in the ICUs to express their wishes to die. Unless there is a will which can be used to express their consent, it is not easy to obtain their consent.

All intensivists agreed that the media allegations were true because in the majority of cases, consent is unavailable.

\section{Question regarding shortening life or hastening the death of patients}

The question was why a medical team would decide to shorten life or hasten the death of a patient and has this been the only option at times.

\section{Responses}

According to intensivist A, if patient is suffering from unbearable pain, with no hope of recovery, hastening the death is sometimes considered and is in fact practiced through titrated dosages of sedative agents and analgesics, to maintain the quality of the dying process. They cannot keep a patient who is dead artificially alive, according to him. He further clarified that sometimes hastening has been the only option.

Intensivist B opinioned that it was where the condition of the patient was hopeless, and all treatments prove to be useless. He was also of the opinion that keeping a patient artificially alive was not beneficial to the patient. Therefore, hastening has sometimes been the only option.

According to intensivist $\mathrm{C}$, when the dignity of the patient is compromised because of futility of treatments, and the patient continues to suffer, hastening the dying process is considered. Hastening is an option when patient suffers unbearable pain with no hope of recovery. Therefore, hastening has sometimes been the only option.

According to intensivist $\mathrm{D}$, painful treatments are considered in determining if hastening the dying process is necessary. For instance, where patient suffers from a treatment such as

Thoracostomy ${ }^{*}+\dagger$ which in itself can be unbearably painful, hastening is considered where the treatment itself does not yield any proportionate benefit. Therefore, hastening has sometimes been the only option.

Finally, according to intensivist E, futility of treatments, unbearable pain and suffering with no hope of recovery, and where the quality of the dying process of the patient is compromised, hastening is considered. Therefore, hastening has been the only option at times, where these factors come into play.

All intensivists were in agreement that futility of treatments, unbearable pain and suffering, with no hope of recovery are the reasons why a medical team would decide to shorten life or hasten the death of a patient. All intensivists were in agreement that shortening life or hastening the death has sometimes been the only option.

\section{Question regarding consent}

The question was whether all patients are incapable of explicit consent and whether there have been exceptions?

\footnotetext{
* Thoracostomy is a medical procedure, where a tube is inserted in the space between the lungs and chest wall (pleural space) to drain fluid, blood, or air from the area around the lungs.

† Thoracotomy is a surgical procedure, where an incision is made to access the chest area to remove a lung, or a piece of a lung, for tests. It is performed typically in patients suffering from lung cancer.
} 


\section{Responses}

According to intensivist A, most patients are incapable of consent because of their critical condition. However, where patient is capable of any communication, the medical team makes a diligent effort to communicate with the patient to procure possible consent to treatment.

This was reiterated by intensivist B, who opined that most patients in the ICUs are unable to communicate and that even if they could, it would normally be very limited communication. It was also made clear by intensivist B that if a patient is able to communicate, and wants a treatment stopped or terminal sedation to alleviate pain and suffering, the medical team must comply with the patient's wishes, in the name of patient autonomy [13]. However, in the majority of cases, it is not possible to obtain consent from the patient.

According to intensivist $\mathrm{C}$, the medical team will always consult with the patient if the patient is able to communicate. In the majority of cases, however, the ICU patient will not be able to communicate his or her wishes at all. Sometimes, they can "agree" or "disagree" based on symbolic speech such as nodding their heads or squeezing the intensivist's hand. Limited communication signaling consent is always possible and is considered an exception in the ICU.

According to intensivist D, while patients' communication certainly depends on the pathology, most patients are incapable of consent in the ICUs.

Finally, according to intensivist E, most patients are incapable of consent. If the patient is able to consent to treatments, the medical team will consult with the patient and will comply with the wishes of the patient. However, the exception, that is, where patient is able to communicate and or consent to treatments, is rare.

All intensivists were in agreement that in the majority of cases, patients in the ICU are incapable of consent. In some exceptional cases, however, they are capable of limited communication to give consent to possible treatments.

\section{Discussion}

Based on the head intensivists' responses gathered from questions on media allegations, hastening and consent, this research study concluded that the media allegations are true. Shortening life or hastening the death does sometimes occur in the ICUs without the non-explicit consent of the patient. The following reasons were given as reasons for this practice, namely:

\section{A. Quality of the dying process of the patient is compromised}

Patient's condition is futile to the point where treatments are not beneficial under the proportionality theory; and too much treatment is not beneficial to the patient and will actually harm the patient under the theory of therapeutic relentlessness [14]. Keeping the patient artificially alive compromises the quality of the dying process of the patient. Hence, shortening life or hastening the death of the patient is only considered when keeping the patient alive compromises the quality of death of the patient [15].

\section{B. Consent is unavailable due to patient's critical condition}

Consent is rendered impossible due to the critical condition of the patient. Where the patient is incapable of explicit consent, the family, or a legal surrogate of the patient is always consulted. In the absence of the family and or legal surrogate, the medical team decides what is in the best interest of the patient and for the benefit of the patient. In arriving at a medical consensus, the medical team consults with specialists trained to treat the patient's particular condition.

During the interviews, there was no ounce of doubt as to the objective of the medical team, where the final decision is to shorten life or hasten the death of the patient [16]. It was in the best interest of the patient, and for the benefit of the patient, not to keep him or her artificially alive.

In conclusion, therefore it was found that while shortening lives or hastening deaths occurred without the nonexplicit consent of the patient, it was not because the medical team did not ask the patient for his or her consent. It was because consent was unavailable due to the patient's critical condition.

\section{Conclusion}

This research study, through its empirical-qualitative research study, investigated whether media allegations that the liberal laws on euthanasia has encouraged doctors to adopt a paternalistic approach towards their patients by terminating their lives without their explicit consent, i.e. engaging in involuntary Euthanasia, were true or false. The research discovered that the media allegations are true, but they are also false. The media allegations are true because shortening life or hastening the death is sometimes practiced in the ICUs without the patient's non-explicit consent. The media allegations are false because consent is not available due to the patient's critical condition, and not 
because it was not asked for. In other words, what is practiced in the ICUs is non-voluntary euthanasia or where patient is unable to request or consent to euthanasia.

\section{Declaration of Competing Interest}

The authors declare that they have no known competing financial interests or personal relationships that could have appeared to influence the work reported in this paper.

\section{Ethical Approval}

All procedures performed in studies involving human participants were in accordance with the ethical standards of the institutional and/or national research committee and with the 1964 Helsinki declaration and its later amendments or comparable ethical standards.

\section{References}

[1] Van Assche K. Recent Belgian Law Reform. Loi-Wet. Available online: http://eol.law.dal.ca/wp-content/uploads/2015/06/Lawof-28-May-2002-on-Euthanasia-as-amended-by-the-Law-of-13-February-2014.pdf (accessed on 16 February 2019).

[2] Nys, H. (2003). A Presentation of the Belgian Act on Euthanasia against the Background of Dutch Euthanasia Law. European Journal of Health Law, 10(3), 239-255. doi:10.1163/157180903770847526.

[3] Patrick J. Hippocrates and Medicine in the Third Millennium. 1997. Available online: http://www.johnpatrick.ca/wpcontent/uploads/2017/02/Hippocrates.pdf (accessed on 16 February 2019).

[4] Kidd RF. The Hippocratic Oath. Welcome to RFKiddcom. Available online: http://rfkidd.com/hippocratic.htm (accessed on 16 February 2019).

[5] Van Zeebroeck, S. (2017). Kill First, Ask Questions Later: The Rule of Law and the Belgian Euthanasia Act of 2002. Statute Law Review, 39(3), 244-257. doi:10.1093/slr/hmx007.

[6] Kidd D, Nys H. The Belgian Act on Euthanasia of May, 28th 2002. Ethical Perspectives 2002. Available online: http://www.ethicalperspectives.be/viewpic.php?TABLE=EP\&ID=59 (accessed on 16 February 2019). p: 182-188.

[7] Jones, D. A., Gastmans, C., \& MacKellar, C. (Eds.). (2017). Euthanasia and assisted suicide: lessons from Belgium (Vol. 42). Cambridge University Press.

[8] Cohen-Almagor, R. (2015). First do no harm: intentionally shortening lives of patients without their explicit request in Belgium. Journal of Medical Ethics, 41(8), 625-629. doi:10.1136/medethics-2014-102387.

[9] Vincent, J.-L., Schetz, M., De Waele, J. J., de Cléty, S. C., Michaux, I., Sottiaux, T., ... Wilmer, A. (2014). "Piece” of mind: End of life in the intensive care unit Statement of the Belgian Society of Intensive Care Medicine. Journal of Critical Care, 29(1), 174-175. doi:10.1016/j.jcrc.2013.08.025.

[10] Cohen-Almagor, R. (2008). Euthanasia policy and practice in Belgium: critical observations and suggestions for improvement. Issues L. \& Med., 24, 187. Available online: https://www.ieb-eib.org/nl/pdf/euthanasia-practice-in-belgium.pdf (accessed on 16 February 2019).

[11] The Belgian Constitution 2007. Available online: https://www.legislationline.org/documents/id/9045 (accessed on 16 February 2019).

[12] Sargeant, J. (2012). Qualitative Research Part II: Participants, Analysis, and Quality Assurance. Journal of Graduate Medical Education, 4(1), 1-3. doi:10.4300/jgme-d-11-00307.1.

[13] Viossat, L.-C. (2019). La loi Claeys-Leonetti sur la fin de vie (France): évaluation de sa mise en œuvre et recommandations pour une meilleure application. Perspectives of Law and Culture on the End-of-Life Legislations in France, Germany, India, Italy and United Kingdom, 45-52. doi:10.5771/9783845296777-45.

[14] Van Zeebroeck, S. (2018). Patient's Orders: Patient's Rights and the Doctor's Obligations under the Claeys-Leonetti Law of 2016 in France. Statute Law Review, 40(3), 266-272. doi:10.1093/slr/hmy004.

[15] Gerson, S. M., Bingley, A., Preston, N., \& Grinyer, A. (2019). When is hastened death considered suicide? A systematically conducted literature review about palliative care professionals' experiences where assisted dying is legal. BMC Palliative Care, 18(1). doi:10.1186/s12904-019-0451-4.

[16] Shapiro, S. P. (2019). Speaking for the Dying: Life-and-Death Decisions in Intensive Care. University of Chicago Press. 